\title{
MicroRNA-34c is regulated by p53 and is involved in sevoflurane-induced apoptosis in the developing rat brain potentially via the mitochondrial pathway
}

\author{
XUE ZHOU $^{1 *}$, DONGFENG XIAN ${ }^{1 *}$, JIEHUA XIA $^{1}$, YING TANG $^{2}$, \\ WENDA LI ${ }^{3}$, XIAOHUI CHEN ${ }^{1}$, ZHIBIN ZHOU ${ }^{1}$, DIHAN LU ${ }^{1}$ and XIA FENG ${ }^{1}$ \\ ${ }^{1}$ Department of Anesthesiology, The First Affiliated Hospital of Sun Yat-sen University; ${ }^{2}$ Department of Anatomy, \\ Zhong Shan School of Medicine, Sun Yat-sen University, Guangzhou, Guangdong 510080; \\ ${ }^{3}$ Department of Hepatobiliary Surgery, The Sun Yat-sen Memorial Hospital of Sun Yat-sen University, \\ Guangzhou, Guangdong 510120, P.R. China
}

Received November 21, 2015; Accepted February 2, 2016

DOI: $10.3892 / \mathrm{mmr} .2017 .6268$

\begin{abstract}
The commonly used inhalation anesthetic, sevoflurane, has been previously demonstrated to induce apoptosis in the developing brain; however, the underlying molecular mechanisms remain largely unknown. MicroRNAs (miRNAs) serve important roles in multiple physiological/pathological processes, such as cell death and survival. In the present study, the miRNA sequence that was most closely associated with sevoflurane-induced apoptosis in the hippocampus of neonatal rat brains was identified. Seven-day-old Sprague Dawley rats were first exposed to $2.3 \%$ sevoflurane for $6 \mathrm{~h}$. Hippocampal brain tissues were harvested at $6 \mathrm{~h}$ following sevoflurane exposure. Cleaved caspase-3 levels were examined using an immunofluorescence assay. Alterations in miRNA expression were assessed by microarray analysis and reverse transcription-quantitative polymerase chain reaction. The protein levels of p53, phosphorylated (p)-p53, B-cell lymphoma-2 (Bcl-2) and Bax were assessed by western blot analysis. Sevoflurane exposure significantly increased the levels of cleaved caspase- 3 in the hippocampus. In addition, among the 688 miRNAs that were observed to be expressed in the hippocampus, sevoflurane exposure altered the expression levels of 266 miRNAs. Among these differentially expressed miRNAs, eight were significantly upregulated and one (miRNA-34c) was significantly downregulated following sevoflurane

Correspondence to: Professor Xia Feng, Department of Anesthesiology, The First Affiliated Hospital of Sun Yat-sen University, 58 Zhongshan 2nd Road, Guangzhou, Guangdong 510080, P.R. China

E-mail: fengxiar@sina.com

*Contributed equally
\end{abstract}

Key words: neurotoxicity, apoptosis, sevoflurane, microRNA, hippocampus exposure. Bioinformatics analyses indicated the miRNA-34c was a direct downstream target of p53. Sevoflurane exposure induced significant alterations in the level of p-p53, Bcl-2 and Bax in the hippocampus of neonatal rats. In conclusion, the results of the present study suggest that miRNA-34c may be regulated by p53 and is involved in sevoflurane-induced neural apoptosis in the hippocampus of developing rat brains, potentially via the mitochondrial pathway.

\section{Introduction}

A large number of newborns and infants are currently treated with anesthetics for surgery in what is considered to be a safe manner. However, accumulating evidence from rodent and nonhuman primate studies, as well as from epidemiological studies (1-5), suggest that exposure to anesthetics during a susceptive neurodevelopmental period may lead to neuronal apoptosis and subsequent learning difficulties (6,7). A previous study demonstrated that sevoflurane exposure led to neuronal apoptosis and pathological alterations in the hippocampus of neonatal rats (8), which was consistent with reports from additional previous studies $(9,10)$. However, the molecular mechanisms underlying sevoflurane-induced neurodegeneration remain largely unknown.

A previous study provided evidence of an association between long-term neurological impairment induced by sevoflurane and alterations in the expression levels of numerous genes in the developing brain (11). MicroRNAs (miRNAs/miRs) are small non-coding RNAs that serve as regulators of endogenous epigenetic gene expression, and serve important roles in multiple physiological/pathological processes, such as cell death and survival $(12,13)$. A recent study demonstrated that sevoflurane combined with propofol anesthesia may lead to alterations in expression levels of miRNAs in the hippocampus of rats (14). In addition, miR-34a has been demonstrated to be involved in ketamine-induced hippocampal apoptosis (15). However, there is limited information regarding the identity of miRNA targets in the hippocampus during and after sevoflurane-induced neural apoptosis. 
miR-34c belongs to the miR-34 family (miRNA-34 a/b/c), which is highly conserved among different species (16). miR-34c is highly expressed in the hippocampus. Targets of $\mathrm{miR}-34 \mathrm{c}$ are considered to be involved in neuronal processes and functions (17). A previous study indicated that miR-34 s are direct transcriptional targets of the p53 tumor suppressor (18). In response to DNA damage, the transcription factor $\mathrm{p} 53$ binds to its consensus binding sequence in promoter DNA, and regulates the expression of miR-34 family genes to induce apoptosis (16). It has been hypothesized that miR-34s complement p53 function through regulation of various targets involved in apoptosis, such as B-cell lymphoma-2 (Bcl-2) (19).

In the present study, the miRNA expression patterns in the hippocampus of rats at $6 \mathrm{~h}$ following exposure to sevoflurane for $6 \mathrm{~h}$, when neural death began to occur, was investigated. In order to determine the functional roles of target genes regulated by altered miR-34c levels, bioinformatics analyses were performed. The results suggested miR-34c may serve a key role in sevoflurane-induced apoptosis, which was the result of p53 phosphorylation, downregulation of $\mathrm{Bcl}-2$ expression and upregulation of Bax.

\section{Materials and methods}

Animals. Experiments were performed using Sprague-Dawley rats (male; age, 7 days; average weight 16-17 g) obtained from the Experimental Animal Center of Sun Yat-sen University, (Guangzhou, China). All interventions and post-anesthesia animal care were performed in accordance with the Guide for the Care and Use of Laboratory Animals (National Research Council, 1996) and the Guidelines of the Institutional Animal Care and Use Committee at Sun Yat-sen University (Guangzhou, China). The use of animals in this study was approved by the Institutional Animal Care and Use Committee at Sun Yat-Sen University (approval no. 2014A-073). All efforts were made to minimize suffering and the number of animals used. The rats were maintained under a 12-h light-dark cycle (light between 07:00 and 19:00), and the room temperature was maintained at $21 \pm 1^{\circ} \mathrm{C}$. Rats had ad libitum access to water and food. A total of 19 litters, 48 control and 51 treated pups were used in the present study.

Sevoflurane exposure. Rats were anesthetized using the protocol described previously (8). Seven-day-old male rats were randomly divided into air-treated control (Con group, $\mathrm{n}=15$ ) and sevoflurane-treated groups (Sevo group, $\mathrm{n}=15$ ). Rats in the Sevo group were placed in a plastic container and exposed to $2.3 \%$ sevoflurane for $6 \mathrm{~h}$ using air as a carrier, with a total gas flow of $2 \mathrm{l} / \mathrm{min}$. In order to maintain the body temperature of rats during sevoflurane exposure, the chamber was heated to $38^{\circ} \mathrm{C}$ using an external heating device (NPS-A3 heated device; Midea Group, Beijiao, China) and an internal hot water bag. The levels of sevoflurane, oxygen and carbon dioxide in the container were monitored using a gas monitor (Detex Ohmeda, Inc., Louisville, CO, USA). During exposure, the respiration frequency and the skin color of the pups was monitored by an investigator. Rats were excluded from the experiment if any signs of apnea or hypoxia were detected. Sevoflurane exposure ceased following $6 \mathrm{~h}$. Upon waking from anesthesia, rats were returned to the maternal cage for
$6 \mathrm{~h}$ before they were sacrificed. The rats in the control group were placed into the same plastic container as those for the sevoflurane group, where they were exposed to air for $6 \mathrm{~h}$.

Arterial blood gas analysis. Arterial blood analysis was performed on both groups using the methods described previously (8). The blood samples were obtained from rats immediately following removal from the maternal cage $(0 \mathrm{~h}, \mathrm{n}=3$ in each subgroup) and at the end of exposure ( $6 \mathrm{~h}, \mathrm{n}=3$ in each subgroup). Briefly, the arterial blood sample (50 $\mu \mathrm{l})$ was rapidly obtained from the left cardiac ventricle and transferred into a heparinized glass capillary tube. Each sample was analyzed immediately following collection with a blood gas analyzer (GEM Premier 3000; Instrumentation Laboratory, Bedford, MA, USA), and the analysis was repeated $>3$ times. The $\mathrm{pH}$, arterial carbon dioxide tension, arterial oxygen tension, and blood glucose levels in arterial blood samples were analyzed in order to exclude other factors, including acidosis, hypercapnia, hypoxemia and hypoglycemia that may induce apoptosis.

Immunofluorescence analysis. In order to determine alterations in the activity of cleaved caspase- 3 in the hippocampus following sevoflurane exposure, rats from the Con and Sevo groups $(n=3)$ were deeply anesthetized with $10 \%$ chloral hydrate (350 mg/kg, Jianglai Biology, Inc., Shanghai, China) intraperitoneally on $\mathrm{P} 7$ at $6 \mathrm{~h}$ following exposure to sevoflurane or air for $6 \mathrm{~h}$, before they were perfused with $4 \%$ paraformaldehyde (Sigma-Aldrich; Merck Millipore, Darmstadt, Germany) in phosphate-buffered saline (PBS; pH 7.4) through the left cardiac ventricle. The brains were dissected and placed in $4 \%$ paraformaldehyde (Sigma-Aldrich; Merck Millipore) at $4^{\circ} \mathrm{C}$ overnight. Following fixation, the brains were soaked in 10, 20 and $30 \%$ sucrose for an additional $24 \mathrm{~h}$. A sliding microtome (Scientific Instruments, Inc., West Palm Beach, FL, USA) was used to cut the brains into coronal sections $(30 \mu \mathrm{m})$, which were blocked with a solution containing $1 \%$ bovine serum albumin (Sigma-Aldrich; Merck Millipore) and $0.4 \%$ Triton X-100 (Sigma-Aldrich; Merck Millipore) for $2 \mathrm{~h}$ at room temperature. Tissue sections were subsequently incubated with a rat anti-rabbit cleaved caspase- 3 primary antibody (cat. no. 9661, dilution, 1:1,500; Cell Signaling Technology, Inc., Danvers, MA, USA) at $4^{\circ} \mathrm{C}$ for $72 \mathrm{~h}$. After washing with PBS, the sections were then incubated with fluorescein isothiocyanate-conjugated anti-rabbit $\operatorname{IgG}$ secondary antibody (cat. no. 43413, dilution, 1:400; Sigma-Aldrich; Merck Millipore) in the dark for $2 \mathrm{~h}$ at room temperature. Finally, the sections were washed with PBS, mounted on gel-coated slides and observed under a fluorescence microscope (Axio Imager Z1; Carl Zeiss AG, Oberkochen, Germany). CA1 and CA3 hippocampal regions in both groups were selected for analysis by fluorescence microscopy. The images were analyzed using Image-Pro Plus software (version, 16.0; Media Cybernetics, Inc., Rockville, MD, USA), and the integral optical density (IOD) of part of the CA1 and CA3 regions in each photograph was collected. A total of 3 sections were analyzed for each rat in both experimental groups $(n=3)$.

Total RNA extraction. Total RNA was isolated from the hippocampus using TRIzol reagent (Invitrogen; Thermo Fisher Scientific, Inc., Waltham, MA, USA), and the miRNeasy Mini 
kit (Qiagen GmbH, Hilden, Germany) according to manufacturer's instructions. The RNA quality and quantity were measured using the NanoDrop ND-1000 spectrophotometer (NanoDrop Technologies; Thermo Fisher Scientific, Inc., Wilmington, DE, USA), and RNA integrity was determined by gel electrophoresis.

miRNA microarray assay and analysis. The microarray assay and analysis of miRNA expression were performed according to the procedures described previously (20). Briefly, the RNA samples from the Con and Sevo groups were labeled using the miRCURY ${ }^{\mathrm{TM}} \mathrm{Hy} 3^{\mathrm{TM}} / \mathrm{Hy}^{\mathrm{TM}}$ Power labeling kit (Exiqon A/S, Vedbaek, Denmark) and hybridized on the miRCURY LNA ${ }^{\mathrm{TM}}$ array (version, 18.0; Exiqon A/S). Following three washes with the wash buffer kit (Exiqon A/S), the slides were scanned using the Agilent G2505C Microarray Scanner system (Agilent Technologies, Inc., Santa Clara, CA, USA), and the images were imported into the Axon GenePix Pro 6.0 software program (Axon Instruments; Molecular Devices, LLC, Sunnyvale, CA, USA) for grid alignment and data extraction. The intensity of the green signal following subtraction of background signal was calculated, and the mean intensity values for replicated spots on the same slide were ascertained to calculate the median intensity for each sample. The following median normalization method was applied to obtain normalized data: (Foreground signal-background signal)/median signal. The median signal was in the 50th percentile of miRNA intensity, and was observed to be $>30$ in all samples following background correction. The threshold value for significance used to define upregulation or downregulation of miRNA expression was considered to be a fold-change of $>1.5$. Hierarchical clustering analysis was performed to demonstrate distinguishable miRNA expression profiles among the samples. A false discovery rate correction was applied for multiple comparisons. miRNAs were selected for further investigation in the present study based on demonstrating a significant different expression pattern when compared with controls (fold-change $>1.5$ ). Target genes of differentially expressed miRNA sequences were identified using Kyoto Encyclopedia of Genes and Genomes (KEGG) pathway analysis.

Reverse transcription-quantitative polymerase chain reaction $(R T-q P C R)$. In order to verify the accuracy of the microarray analysis, RT-qPCR experiments were performed to determine the expression of selected miRNAs using the procedures described in the manufacturer's protocol (Takara Biotechnology, Co., Ltd., Dalian, China). The primers (Invitrogen; Thermo Fisher Scientific, Inc.) used are shown in Table I. The RNA extracts were reverse transcribed into cDNA. In specific, the MMLV reverse transcriptase enzyme (Takara Biotechnology, Co., Ltd.) and RT primers of U6 and rno-miR-34c were used. The RT-qPCR reaction was performed at $95^{\circ} \mathrm{C}$ for $10 \mathrm{~min}$ (pre-denaturation) followed by 42 cycles of $95^{\circ} \mathrm{C}$ for $15 \mathrm{sec}, 58^{\circ} \mathrm{C}$ for $30 \mathrm{sec}$ and $72^{\circ} \mathrm{C}$ for $30 \mathrm{sec}$. Following the reaction, a melting curve analysis from 55 to $95^{\circ} \mathrm{C}$ was applied to all reactions to ensure the consistency and specificity of the amplified product. The data were analyzed using the $\mathrm{iQ}^{\mathrm{TM}} 5$ Optical System software (Bio-Rad Laboratories, Inc., Hercules, CA, USA). The RT-qPCR data were normalized using U6 RNA. The relative miRNA expression was determined by calculating the mean difference between the cycle thresholds of the miRNA and the U6 control for each sample $\left[\Delta\right.$ quantification cycle $\left.\left(\Delta \mathrm{C}_{\mathrm{q}}\right)\right]$ within each sample group, which was expressed as $-\Delta \mathrm{C}_{\mathrm{q}}$ for the relative change in expression. The fold-change in miRNA expression was determined by calculating the difference between the mean $\Delta \mathrm{C}_{\mathrm{q}}$ of the Sevo and Con groups at the same time point and hippocampal location $\left(\Delta \Delta \mathrm{C}_{\mathrm{q}}\right)$, and this value was expressed as the fold-change $\left(2^{-\Delta \Delta \mathrm{Cq})}(21)\right.$. The difference in cycle threshold alterations $\left(-\Delta \Delta \mathrm{C}_{\mathrm{q}}\right)$ was determined using a Student's two-tailed t-test, and a $\mathrm{P}<0.05$ was considered to indicate a statistically significant difference.

miRNA target gene prediction and functional analysis. Using the Gene Ontology project (http://www.geneontology.org/), target genes that were affected by the differentially expressed miRNAs in sevoflurane-exposed rat pups were identified. The Gene Ontology project was used to perform cluster analysis of the enriched biological themes, and enrichment scores equal to - $\log 10$ (P-value) were considered to indicate potential pathways. Higher enrichment score values indicate a low P-value, which therefore indicates that the cluster is significant.

Western blot analysis. At $6 \mathrm{~h}$ following exposure to sevoflurane or air for $6 \mathrm{~h}, 3$ rats in each group were sacrificed by decapitation. The brain was dissected, and the hippocampus was quickly removed. The tissue samples were homogenized in $100 \mathrm{mg} / \mathrm{ml}$ radioimmunoprecipitation assay lysis buffer (Shanghai Shenergy Biocolor BioScience \& Technology Company, Shanghai, China) containing $1 \%$ (v/v) phenylmethylsulfonyl fluoride (Shanghai Shenergy Biocolor BioScience \& Technology Company) and centrifuged at 13,000 x $g$ for $20 \mathrm{~min}$ at $4^{\circ} \mathrm{C}$. The supernatant was separated, and the quantity of total protein extracted was measured using a Bio-Rad Protein assay (Bio-Rad Laboratories, Inc.). The samples were boiled, loaded at a concentration of $50 \mathrm{mg}$ sample/lane and electrophoresed on a 10\% SDS-PAGE gel, and transferred onto polyvinylidene fluoride membranes (Pall Life Sciences, Port Washington, NY, USA). The blotted membranes were incubated with rabbit polyclonal anti-p53 (dilution, 1:2,000; cat. no. Asp175; Cell Signaling Technology, Inc.), rabbit polyclonal anti-phosphorylated (p)-p53 (dilution, 1:3,000; cat. no. Asp175; Cell Signaling Technology, Inc.), mouse monoclonal anti-Bcl-2 (dilution, 1:1,000; cat. no. 15071; Cell Signaling Technology, Inc.), mouse monoclonal anti-Bax (dilution, 1:1,500; cat. no. 2772; Cell Signaling Technology, Inc.), mouse monoclonal anti- $\beta$-actin (dilution, 1:2,000; cat. no. sc-58673; Santa Cruz Biotechnology, Inc., Dallas, TX, USA) or rabbit polyclonal anti-cleaved caspase-3 (dilution, 1:1,000; cat. no. 9664; Cell Signaling Technology, Inc.) antibodies overnight at room temperature. The membranes were then washed three times with PBS+0.05\% Tween-20 (Sigma-Aldrich; Merck Millipore) mixture, and incubated for $2 \mathrm{~h}$ at room temperature with the anti-rabbit or anti-mouse IgG horseradish peroxidase-conjugated secondary antibodies (dilution, 1:2,000; cat. no. sc-2372 or sc-2377; Santa Cruz Biotechnology, Inc.). The protein expression levels were examined using the Pierce ECL Plus Western Blotting Substrate system (Pierce; Thermo Fisher Scientific, Inc.) and photographed. The optical density was measured using ImageJ software, V1.48u (National Institutes 
Table I. Primers used for RT and RT-qPCR analysis of the miRNA expression.

\begin{tabular}{|c|c|c|}
\hline Procedure & Name & Sequence $\left(5^{\prime}-3^{\prime}\right)$ \\
\hline \multirow[t]{10}{*}{ RT } & U6 & CGCTTCACGAATTTGCGTGTCAT \\
\hline & $\operatorname{miR}-3559-5 p$ & GTCGTATCCAGTGCGTGTCGTGGAGTCGGCAATTGCACTGGATACGACTCATGTAG \\
\hline & miR-92a-2-5p & GTCGTATCCAGTGCGTGTCGTGGAGTCGGCAATTGCACTGGATACGACGTAATG \\
\hline & $\operatorname{miR}-214-5 p$ & GTCGTATCCAGTGCGTGTCGTGGAGTCGGCAATTGCACTGGATACGACAGACAC \\
\hline & $\operatorname{miR}-382-3 p$ & GTCGTATCCAGTGCGTGTCGTGGAGTCGGCAATTGCACTGGATACGACAAGTGT \\
\hline & $\operatorname{miR}-181 b-1-3 p$ & GTCGTATCCAGTGCGTGTCGTGGAGTCGGCAATTGCACTGGATACGACTTGCAT \\
\hline & $\operatorname{miR}-101 a-5 p$ & GTCGTATCCAGTGCGTGTCGTGGAGTCGGCAATTGCACTGGATACGACGCATCAG \\
\hline & $\operatorname{miR}-130 b-5 p$ & GTCGTATCCAGTGCGTGTCGTGGAGTCGGCAATTGCACTGGATACGACGTAGTG \\
\hline & $\operatorname{miR}-188-3 p$ & GTCGTATCCAGTGCGTGTCGTGGAGTCGGCAATTGCACTGGATACGACGCAAAC \\
\hline & $\operatorname{miR}-34 c-5 p$ & GTCGTATCCAGTGCGTGTCGTGGAGTCGGCAATTGCACTGGATACGACGCAATC \\
\hline \multirow[t]{18}{*}{ RT-qPCR } & U6 & F: GCTTCGGCAGCACATATACTAAAAT \\
\hline & & R: CGCTTCACGAATTTGCGTGTCAT \\
\hline & $\operatorname{miR}-3559-5 p$ & GSP: GGGGGGGTGACAGACTTAGTA \\
\hline & miR-92a-2-5p & GSP: GGAAGGTGGGGATTAGTGC \\
\hline & & R: GTGCGTGTCGTGGAGTCG \\
\hline & miR-214-5p & $\begin{array}{l}\text { GSP: GGGGGGAAAGAGTTGTCATG } \\
\text { R : GTGCGTGTCGTGGAGTCG }\end{array}$ \\
\hline & miR-382-3p & GSP: GGGCAATCATTCACGGACA \\
\hline & & R: GTGCGTGTCGTGGAGTCG \\
\hline & miR-181b-1-3p & GSP: GGGGGCTCACTGAACAATG \\
\hline & & R: GTGCGTGTCGTGGAGTCG \\
\hline & $\operatorname{miR}-101 a-5 p$ & GSP: GGGGGGTCAGTTATCACAGTG \\
\hline & & R: GTGCGTGTCGTGGAGTCG \\
\hline & miR-130b-5p & GSP: GCCACTCTTTCCCTGTTG \\
\hline & & R: CAGTGCGTGTCGTGGAG \\
\hline & miR-188-3p & GSP: GGAATCTCCCACATGCAGG \\
\hline & & R: GTGCGTGTCGTGGAGTCG \\
\hline & $\operatorname{miR}-34 c-5 p$ & GSP: GGGAGGCAGTGTAGTTAGC \\
\hline & & R: CAGTGCGTGTCGTGGAGT \\
\hline
\end{tabular}

RT-qPCR, reverse transcription-quantitative polymerase chain reaction; miR, microRNA; F, forward; GSP, gene-specific primer; R, reverse.

of Health, Bethesda, MD, USA). Immunoblots were performed at least three times for each hippocampus sample from each rat.

Statistical analysis. The data are presented as the mean \pm standard deviation. Statistical analysis was performed using the two-tailed Student's t-test and the SPSS software program (version, 16.0; SPSS, Inc., Chicago, IL, USA). All data in the study represent at least three independent experiments for 3 samples in each experiment. $\mathrm{P}<0.05$ was considered to indicate a statistically significant difference.

\section{Results}

Sevoflurane exposure did not alter the parameters of arterial blood analysis. As shown in Table II, no significant alterations were observed between the control group and the sevoflurane group for $\mathrm{pH}$, oxygen tension, carbon dioxide tension and glucose levels. During sevoflurane and air exposure, the skin color of the rat pups remained pink (data not shown).
Sevoflurane exposure increased the levels of cleaved caspase-3 in the hippocampus. In order to determine the effect of sevoflurane exposure on apoptosis in hippocampal tissues, immunohistochemistry assay analysis was performed to measure the level of cleaved caspase-3 (red regions; Fig. 1A and B) and the IOD of CA1 and CA3 regions. As illustrated in Fig. 1C, the IODs in the CA1 and CA3 regions were significantly higher in the sevoflurane group when compared with the control group $(\mathrm{P}=0.001)$.

Sevoflurane exposure altered miRNA expression in the hippocampus. Microarray analysis indicated that 688 miRNAs were expressed in the hippocampus of rats at 7 days of age. Following normalization of the signal intensities to the miRNA expression levels, miR-124-3p, miR-9a-3p and miR-125b-5p were observed to be expressed at the highest levels (Fig. 2). Compared with the control group, the expression levels of 266 miRNAs in the hippocampus of the sevoflurane group were altered. Among these 266 miRNAs, 8 miRNAs (miR-3559-5p, 
Table II. Arterial blood analysis.

\begin{tabular}{lcccccc}
\hline Group & Time $(\mathrm{h})$ & $\mathrm{n}$ & $\mathrm{pH}$ & $\mathrm{PaCO}_{2}(\mathrm{Kpa})$ & $\mathrm{PaO}_{2}(\mathrm{Kpa})$ & $\mathrm{Glucose}(\mathrm{mmol} / \mathrm{l})$ \\
\hline Control & 0 & 3 & $7.38 \pm 0.04$ & $3.55 \pm 0.44$ & $13.33 \pm 0.23$ & $5.4 \pm 0.6$ \\
& 6 & 3 & $7.37 \pm 0.06$ & $3.56 \pm 0.14$ & $13.32 \pm 0.51$ & $5.3 \pm 0.8$ \\
Sevoflurane & 0 & 3 & $7.38 \pm 0.03$ & $3.56 \pm 0.29$ & $13.35 \pm 0.25$ & $5.5 \pm 0.3$ \\
& 6 & 3 & $7.38 \pm 0.02$ & $3.57 \pm 0.54$ & $13.32 \pm 0.61$ & $5.3 \pm 0.6$ \\
\hline
\end{tabular}

Neonatal exposure to sevoflurane did not induce significant cardiorespiratory dysfunction. Analysis of arterial blood gas indicated no significant differences in any of the parameters between rats exposed for $6 \mathrm{~h}$ to sevoflurane and the control group exposed to air for $6 \mathrm{~h}(\mathrm{P}>0.05)$. $\mathrm{PaCO}$, arterial carbon dioxide tension; $\mathrm{PaO}_{2}$, arterial oxygen tension.
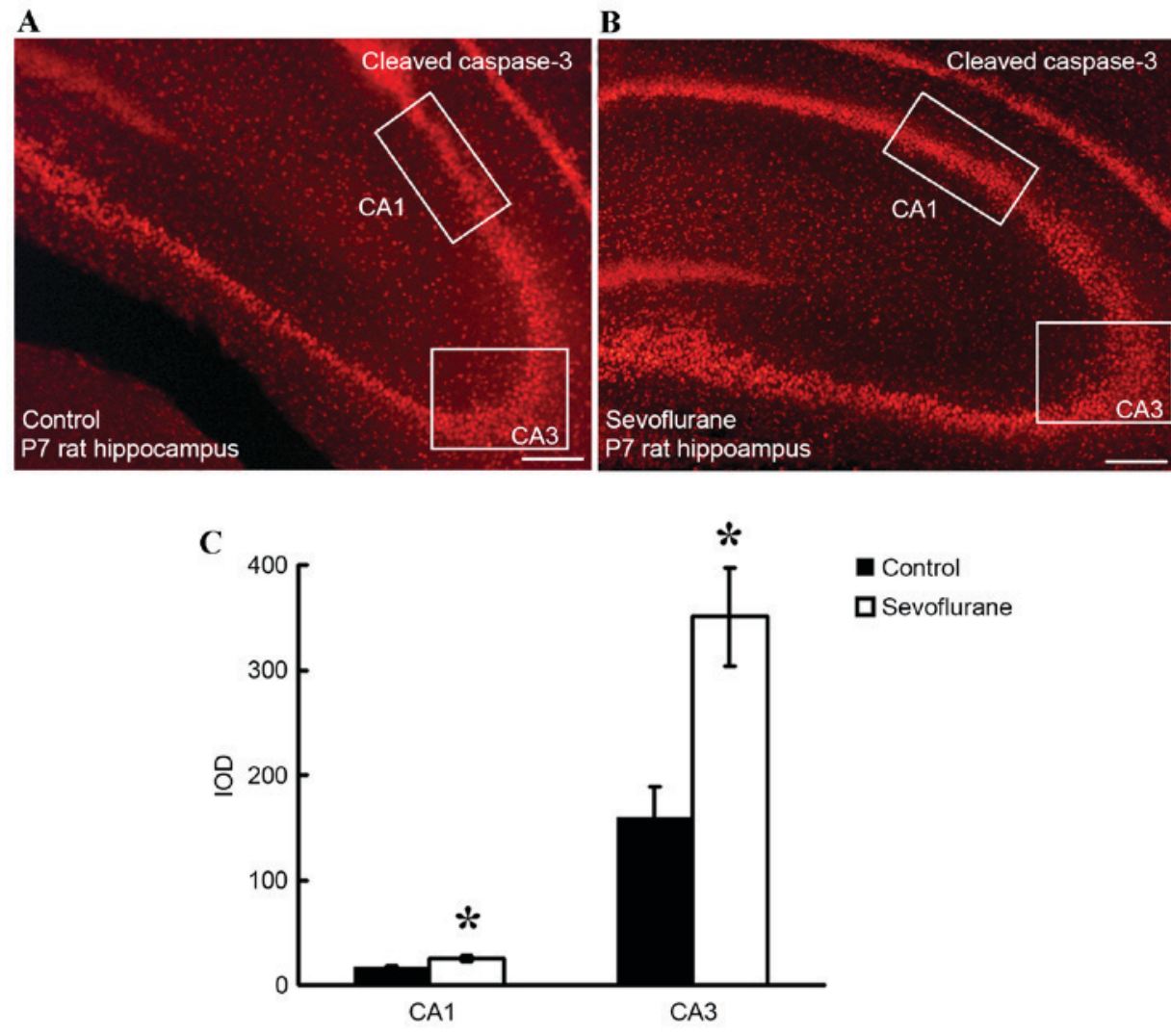

Figure 1. Sevoflurane exposure increases the level of cleaved caspase-3 in the hippocampus. Immunofluorescence staining of cleaved caspase-3 (red) in the hippocampus at $6 \mathrm{~h}$ following exposure to (A) air or (B) sevoflurane for $6 \mathrm{~h}$ (scale bar, $50 \mu \mathrm{m}$ ). (C) The IODs in the CA1 and CA3 hippocampal regions of control group and sevoflurane group rats were shown. ${ }^{*} \mathrm{P}<0.05$ vs. control. IOD, integral optical density.

miR-92a-2-5p, miR-214-5p, miR-382-3p, miR-181b-1-3p, miR-101a-5p, miR-130b-5p, and miR-188-3p) were significantly upregulated, and one miRNA (miR34-c) was significantly downregulated following sevoflurane exposure $(\mathrm{P}=0.001$; Fig. 3A).

Sevoflurane exposure induced alterations in miRNA expression as determined by identified by RT-qPCR analysis. In order to validate the microarray results, RT-qPCR analysis was performed to confirm the expression of the nine identified miRNAs. As demonstrated in Fig. 3B, RT-qPCR analysis confirmed that, miR-3559-5p, miR-92a-2-5p, miR-214-5p, miR-382-3p, miR-181b-1-3p, miR-101a-5p, miR-130b-5p and miR-188-3p expression levels were increased, whereas
miR34-c expression was significantly reduced following sevoflurane exposure.

Bioinformatics analyses of the miRNA targets. Target genes of differentially expression miRNA sequences were identified using KEGG pathway analysis (http://www.genome.jp/kegg/). All pathways that demonstrated a P-value of $<0.05$ are listed in Table III. The identified pathways included mineral absorption, tuberculosis, the p53 signaling pathway, glycolysis/gluconeogenesis, drug metabolism-cytochrome P450, metabolism of cytochrome by cytochrome $\mathrm{P} 450$, antigen processing and presentation, and pyrimidine metabolism associated genes. Among these pathways, only miR-34c was directly associated with the p53 signaling pathway. This suggests that the 


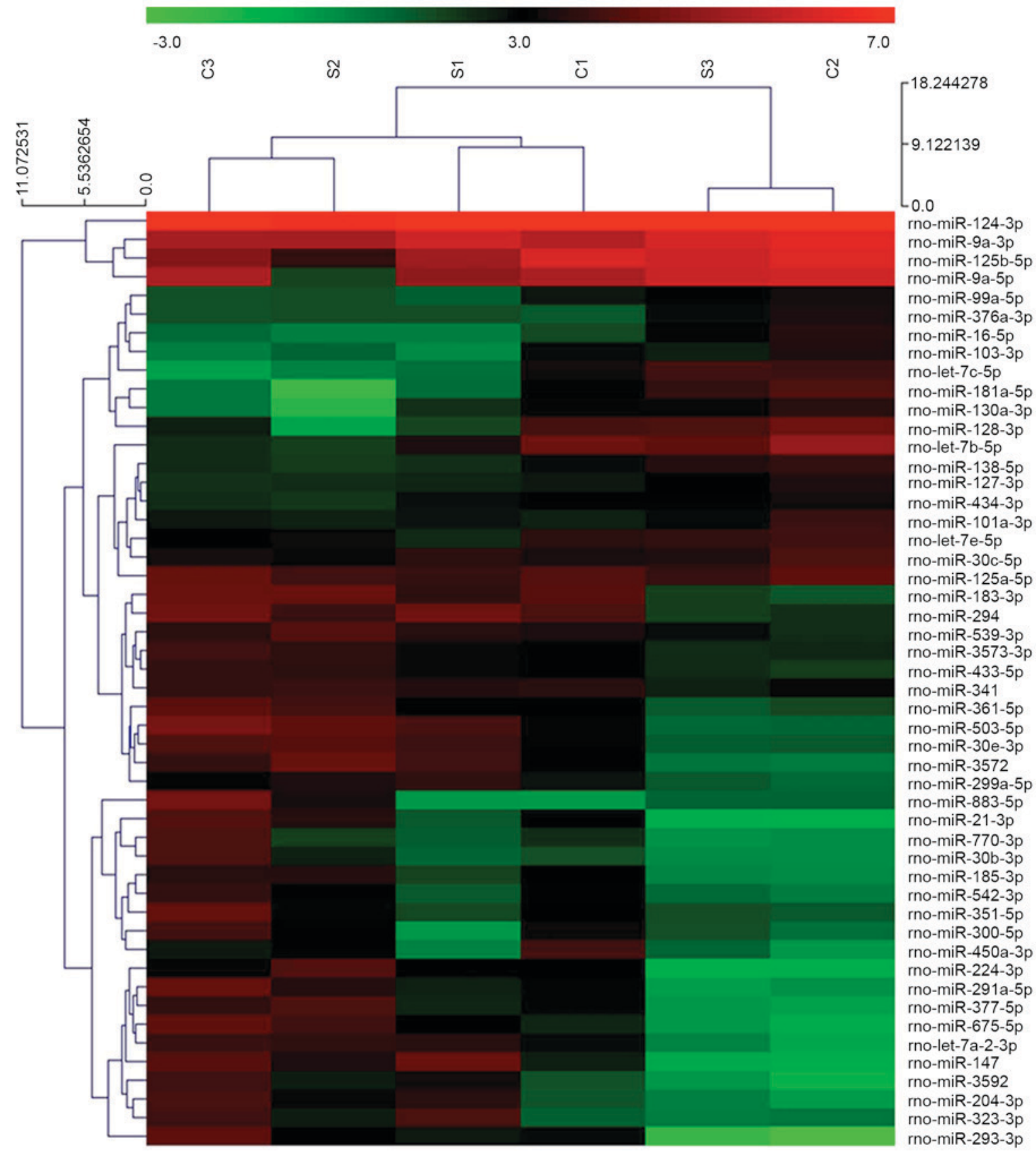

Figure 2. miRNA expression profiles at $6 \mathrm{~h}$ following exposure to air or sevoflurane for $6 \mathrm{~h}$. The heat map consisted of a collection of the 50 most highly expressed miRNAs, with a signal intensity of $>30$ in rats that were exposed to air or $2.3 \%$ sevoflurane for $6 \mathrm{~h}$ before they were sacrificed at $6 \mathrm{~h}$ following exposure. Red indicates a relatively high expression level, and green indicates a relatively low expression level. miRNA, microRNA; C1, C2 and C3, control samples; S1, S2 and S3, sevoflurane samples.

miR-34c and the p53 pathway may be involved in mediating sevoflurane-induced apoptosis in the hippocampus of developing rat brains.

Sevoflurane altered the levels of p53, Bcl-2, Bax and cleaved caspase-3 in the hippocampus of the neonate rat brain. Western blot analysis was performed to assess the effect of sevoflurane on the targets of the p53 signaling pathway in the hippocampus. As shown in Fig. 4, sevoflurane exposure significantly increased the level of the p-p53 protein $(\mathrm{P}=0.03$; Fig. 4A) and $\mathrm{Bax}(\mathrm{P}=0.001$; Fig. 4B), and significantly decreased the level of Bcl-2 protein $(\mathrm{P}=0.001$; Fig. $4 \mathrm{~B})$ in the hippocampus of neonate rat brains when compared with the control rat brains. In addition, sevoflurane exposure significantly increased the expression of cleaved caspase- 3 when compared with the control group $(\mathrm{P}<0.05$; Fig. $4 \mathrm{C})$.

\section{Discussion}

In the present study, arterial blood analysis was first performed in rats exposed to sevoflurane or air, which confirmed that none of the pups in either group had suffered from apnea or hypoxia during treatment. The level of apoptosis in the hippocampus of neonatal rats at $6 \mathrm{~h}$ following sevoflurane exposure for $6 \mathrm{~h}$ was then examined. Although apoptosis is known to occur in the developing brain (22), it was more evident following exposure 
Table III. Signaling pathways identified by Kyoto Encyclopedia of Genes and Genomes pathway analysis in rats at $6 \mathrm{~h}$ following exposure to sevoflurane for $6 \mathrm{~h}$.

\begin{tabular}{lrr}
\hline Pathway & Count & P-value \\
\hline Mineral absorption & 46 & 0.008190094 \\
Tuberculosis & 193 & 0.01902255 \\
p53 signaling pathway & 75 & 0.02084327 \\
Glycolysis/gluconeogenesis & 79 & 0.02297618 \\
Drug metabolism-cytochrome P450 & 85 & 0.02633797 \\
Metabolism of xenobiotics by cytochrome P450 & 89 & 0.0286843 \\
Antigen processing and presentation & 94 & 0.03173175 \\
Pyrimidine metabolism & 100 & 0.03555134
\end{tabular}
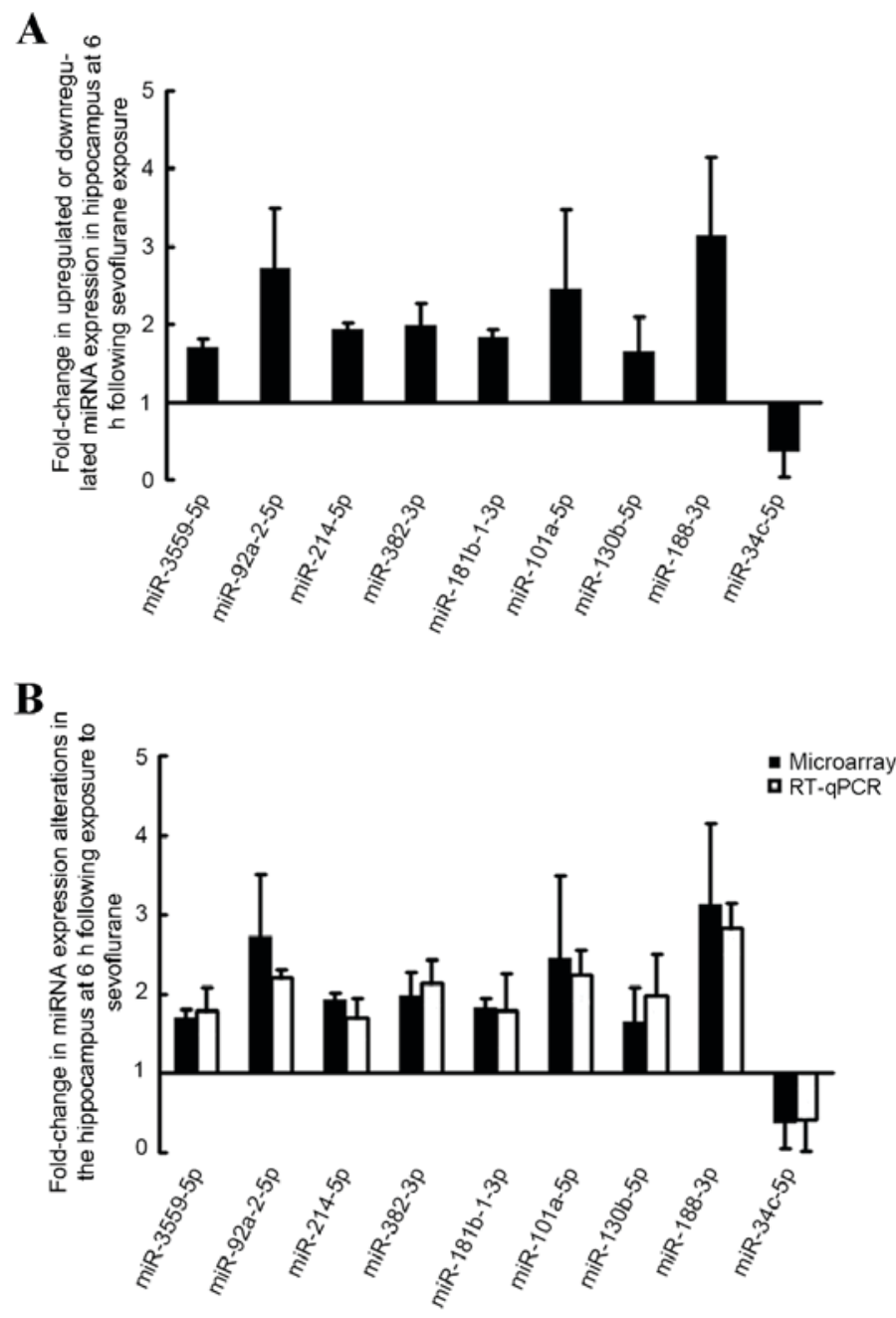

Figure 3. Significant expression alterations in miRNAs at $6 \mathrm{~h}$ following exposure to sevoflurane for $6 \mathrm{~h}$. (A) Significantly upregulated and downregulated miRNAs. (B) The fold-change in miRNA expression as determined by reverse transcription-quantitative polymerase chain reaction and microarray analyses. miRNA, microRNA.

to $2.3 \%$ sevoflurane for $6 \mathrm{~h}$, as indicated by the significant increase in the level of cleaved caspase-3; a marker of the execution phase of cell apoptosis (23). This result is consistent with studies conducted in our lab previously $(8,24)$. Additional studies involving a variety of adolescent animal models have demonstrated that sevoflurane induces apoptosis, which may contribute to the deficits in hippocampus-dependent learning and memory $(9,10,25)$. However, the detailed mechanisms underlying the harmful effects of sevoflurane in the developing brain are not fully understood.

miRNAs serve key roles in a wide range of biological processes, including cell death and survival, as well as regulation of gene expression, which occurs primarily at the translational level $(12,13)$. The results of the current study 
A

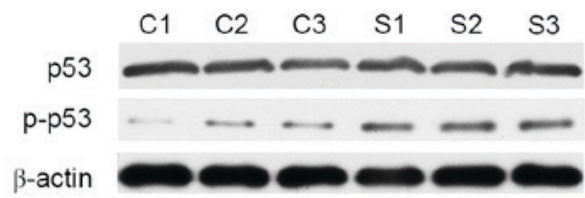

B

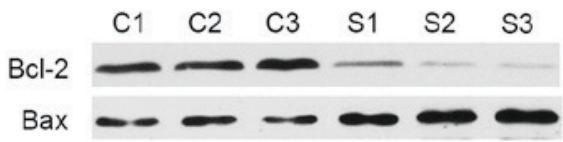

C

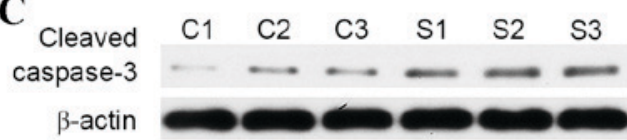

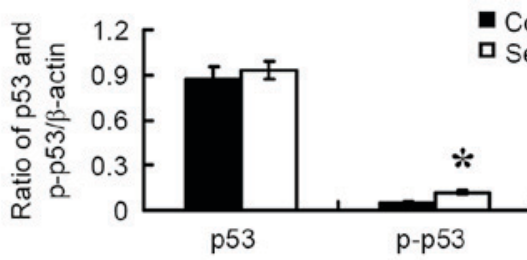
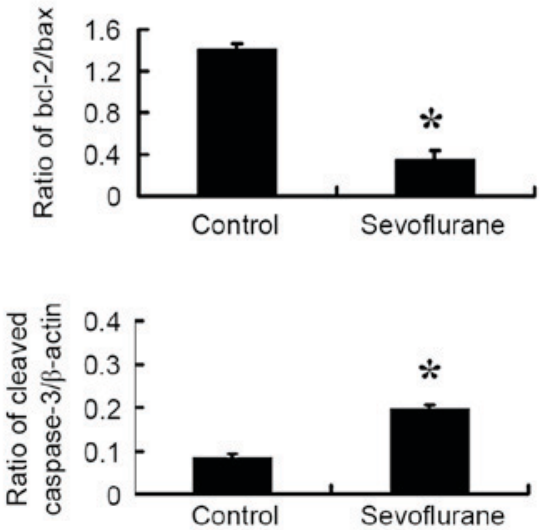

Figure 4. Western blot analysis demonstrated that exposure to sevoflurane for $6 \mathrm{~h}$ induced a significant increase in p-p53, Bax and cleaved caspase-3 protein levels, and a significant decrease in Bcl-2 protein levels when compared with controls. Representative graphs showing the protein expression levels of (A) p53 and p-p53, (B) Bcl-2 and Bax (Bcl-2/Bax ratio is used to determine apoptosis), and (C) cleaved caspase-3. Samples were obtained from the hippocampus of rats exposed to air (C1-C3) or sevoflurane (S1-S3). ${ }^{*} \mathrm{P}<0.05$ vs. control. p-p53, phosphorylated p-53; Bcl-2, B-cell lymphoma-2.

provide novel evidence to suggest that exposure to $2.3 \%$ sevoflurane for $6 \mathrm{~h}$ affects miRNA expression patterns in the hippocampus. Hierarchical clustering analysis, presented as a heat map, was performed to visualize the semantic similarities and expression levels of the miRNAs. The results demonstrated that miR-124-3p, miR-9a-3p and miR-125b-5p were expressed at the highest levels. Following sevoflurane exposure, 8 miRNAs were significantly upregulated, while only miR-34c was significantly downregulated. Therefore, miR34c was selected for further investigation. Notably, Goto et al (14) reported a contrasting result. In this previous study, 4 miRNAs were observed to be significantly increased, while 12 miRNAs were significantly reduced in the rat hippocampus following sevoflurane anesthesia. The reasons underlying the difference between their results and those of the present study remain to be elucidated. However, one notable difference is that Goto et al (14) conducted experiments on 6-week-old male Wistar rats, whereas 7-day-old Sprague-Dawley rats were employed in the current study. Previous studies have demonstrated that ketamine leads to neuroapoptosis in the developing brain (26), and that miR-34a negatively regulates ketamine-induced apoptosis in the hippocampus (15). This is particularly notable, as miR-34a and miR-34c belong to the miR-34 family. In addition, previous studies have indicated that the miR-34 family is associated with additional biological processes including cancer (27); however its role in the developing brain has been rarely addressed. It has been reported that miR-34 family genes are involved in apoptosis (19), and the results of the present study suggest that miR-34c may be involved in apoptosis induced by sevoflurane.

miR-34 family members are direct transcriptional targets of the p53 tumor suppressor (18). Previous studies have demonstrated that $\mathrm{p} 53$ regulates the expression of miR-34c $(16,28)$. KEGG is a compendium of databases containing annotated genomes and protein interaction networks for all organisms that have undergone genome sequencing, as well as a compilation of manually verified pathway maps displaying molecular interactions and biochemical reactions (29). Using KEGG pathway analysis in the current study, several pathways were observed to be significantly correlated to the present condition (sevoflurane-treated P7 rats), including the p53 pathway, and p53 phosphorylation was significantly increased. The authors hypothesize that sevoflurane may lead to activation of p53 and the downstream target, miR-34c. Among all the predicted pathways identified, p53 regulates apoptosis, in part, by inducing intrinsic cell death (30). The intrinsic, mitochondrial pathway is regulated by Bcl-2 family proteins, including the anti-apoptotic factor Bcl-2 and the pro-apoptotic factor Bax (31). Recent studies have indicated that a number of miRNAs directly target $\mathrm{Bcl}-2$ family proteins including miRNA-34 (32). The results of the present study indicated that Bax protein levels were significantly increased, while Bcl-2 protein levels were significantly decreased. These results suggest that miR-34c may be regulated by activated p53, and is involved in sevoflurane-induced apoptosis in the developing brain potentially via the mitochondrial pathway. These results are consistent with a previous study, which demonstrated that sevoflurane induces caspase-dependent, mitochondria-mediated apoptosis in human T lymphocytes in vitro (33).

There are several limitations of the current study. Firstly, the expression of additional miRNAs, aside from miR-34c, was observed to be significantly altered following sevoflurane exposure. It remains formally possible that these miRNAs may contribute to the apoptosis induced by sevoflurane. Secondly, miRNA-34c is transactivated by $\mathrm{p} 53$; however, there is insufficient evidence to exclude the possibility that additional miRNAs may be involved in the p53 signaling pathway. Further research will be designed and performed to 
address these issues. For example, by conducting interference of miR-34 expression in vivo or in vitro, in order to identify the precise role of miR-34c in sevoflurane-induced apoptosis. Furthermore, additional pathways may be involved in sevoflurane-induced apoptosis, and these mechanisms warrant further investigation.

In conclusion, exposure to $2.3 \%$ sevoflurane for $6 \mathrm{~h}$ led to apoptosis in the developing rat hippocampus. The results of the present study suggest that miR-34c may be regulated by $\mathrm{p} 53$, and may be involved in sevoflurane-induced neural apoptosis. Sevoflurane affects Bax and Bcl-2 levels and induces apoptosis potentially via the mitochondrial pathway in the hippocampus of the developing rat brains.

\section{Acknowledgements}

The present study was supported by the National Science Foundation Council of China (81571032).

\section{References}

1. Wilder RT, Flick RP, Sprung J, Katusic SK, Barbaresi WJ, Mickelson C, Gleich SJ, Schroeder DR, Weaver AL and Warner DO: Early exposure to anesthesia and learning disabilities in a population-based birth cohort. Anesthesiology 110: 796-804, 2009

2. DiMaggio C, Sun LS and Li G: Early childhood exposure to anesthesia and risk of developmental and behavioral disorders in a sibling birth cohort. Anesth Analg 113: 1143-1151, 2011.

3. Ing C, DiMaggio C, Whitehouse A, Hegarty MK, Brady J, von Ungern-Sternberg BS, Davidson A, Wood AJ, Li G and Sun LS Long-term differences in language and cognitive function after childhood exposure to anesthesia. Pediatrics 130: e476-e485, 2012.

4. Hansen TG, Pedersen JK, Henneberg SW, Pedersen DA, Murray JC, Morton NS and Christensen K: Academic performance in adolescence after inguinal hernia repair in infancy: A nationwide cohort study. Anesthesiology 114: 1076-1085, 2011.

5. Sun L: Early childhood general anaesthesia exposure and neurocognitive development. Br J Anaesth 105 (Suppl 1): i61-i68, 2010.

6. Deng M, Hofacer RD, Jiang C, Joseph B, Hughes EA, Jia B, Danzer SC and Loepke AW: Brain regional vulnerability to anaesthesia-induced neuroapoptosis shifts with age at exposure and extends into adulthood for some regions. $\mathrm{Br} \mathbf{J}$ Anaesth 113: 443-451, 2014

7. Zou X, Liu F, Zhang X, Patterson TA, Callicott R, Liu S, Hanig JP, Paule MG, Slikker W Jr and Wang C: Inhalation anesthetic-induced neuronal damage in the developing rhesus monkey. Neurotoxicol Teratol 33: 592-597, 2011.

8. Feng X, Liu JJ, Zhou X, Song FH, Yang XY, Chen XS, Huang WQ, Zhou LH and Ye JH: Single sevoflurane exposure decreases neuronal nitric oxide synthase levels in the hippocampus of developing rats. Br J Anaesth 109: 225-233, 2012.

9. Zhang X, Xue Z and Sun A: Subclinical concentration of sevoflurane potentiates neuronal apoptosis in the developing C57BL/6 mouse brain. Neurosci Lett 447: 109-114, 2008.

10. Satomoto M, Satoh Y, Terui K, Miyao H, Takishima K, Ito M and Imaki J: Neonatal exposure to sevoflurane induces abnormal social behaviors and deficits in fear conditioning in mice. Anesthesiology 110: 628-637, 2009.

11. Pan ZQ, Lu XF, Shao C, Zhang C, Yang J, Ma T, Zhang LC and Cao JL: The effects of sevoflurane anesthesia on rat hippocampus: A genomic expression analysis. Brain Res 1381: 124-133, 2011.

12. Carthew RW and Sontheimer EJ: Origins and mechanisms of miRNAs and siRNAs. Cell 136: 642-655, 2009.

13. Moreno-Moya JM, Vilella F and Simón C: MicroRNA: Key gene expression regulators. Fertil Steril 101: 1516-1523, 2014.
14. Goto G, Hori Y, Ishikawa M, Tanaka S and Sakamoto A: Changes in the gene expression levels of microRNAs in the rat hippocampus by sevoflurane and propofol anesthesia. Mol Med Rep 9: 1715-1722, 2014.

15. Jiang XL, Du BX, Chen J, Liu L, Shao WB and Song J: MicroRNA-34a negatively regulates anesthesia-induced hippocampal apoptosis and memory impairment through FGFR1. Int J Clin Exp Pathol 7: 6760-6767, 2014.

16. He L, He X, Lim LP, de Stanchina E, Xuan Z, Liang Y, Xue W, Zender L, Magnus J, Ridzon D, et al: A microRNA component of the p53 tumour suppressor network. Nature 447: 1130-1134, 2007.

17. Zovoilis A, Agbemenyah HY, Agis-Balboa RC, Stilling RM, Edbauer D, Rao P, Farinelli L, Delalle I, Schmitt A, Falkai P, et al: microRNA-34c is a novel target to treat dementias. Embo J 30: 4299-4308, 2011

18. Cha YH, Kim NH, Park C, Lee I, Kim HS and Yook JI: MiRNA-34 intrinsically links p53 tumor suppressor and Wnt signaling. Cell Cycle 11: 1273-1281, 2012.

19. Suzuki HI and Miyazono K: Dynamics of microRNA biogenesis: Crosstalk between p53 network and microRNA processing pathway. J Mol Med (Berl) 88: 1085-1094, 2010.

20. Tang Y, Ling ZM, Fu R, Li YQ, Cheng X, Song FH, Luo HX and Zhou LH: Time-specific microRNA changes during spinal motoneuron degeneration in adult rats following unilateral brachial plexus root avulsion: Ipsilateral vs. contralateral changes. BMC Neurosci 15: 92, 2014.

21. Livak KJ and Schmittgen TD: Analysis of relative gene expression data using real-time quantitative PCR and the 2(-Delta Delta C (T)) Method. Methods 25: 402-408, 2001.

22. Yudkowitz FS: Anesthetics and the developing brain. Semin Cardiothorac Vasc Anesth 14: 44-45, 2010.

23. Lemkuil BP, Head BP, Pearn ML, Patel HH, Drummond JC and Patel PM: Isoflurane neurotoxicity is mediated by p75 (NTR)-RhoA activation and actin depolymerization. Anesthesiology 114: 49-57, 2011.

24. Zhou X, Song FH, He W, Yang XY, Zhou ZB, Feng X and Zhou LH: Neonatal exposure to sevoflurane causes apoptosis and reduces nNOS protein expression in rat hippocampus. Mol Med Rep 6: 543-546, 2012.

25. Haseneder R, Kratzer S, von Meyer L, Eder M, Kochs E and Rammes G: Isoflurane and sevoflurane dose-dependently impair hippocampal long-term potentiation. Eur J Pharmacol 623: 47-51, 2009.

26. Brambrink AM, Evers AS, Avidan MS, Farber NB, Smith DJ, Martin LD, Dissen GA, Creeley CE and Olney JW: Ketamine-induced neuroapoptosis in the fetal and neonatal rhesus macaque brain. Anesthesiology 116: 372-384, 2012.

27. Maroof H, Salajegheh A, Smith RA and Lam AK: Role of microRNA-34 family in cancer with particular reference to cancer angiogenesis. Exp Mol Pathol 97: 298-304, 2014.

28. Corney DC, Flesken-Nikitin A, Godwin AK, Wang W and Nikitin AY: MicroRNA-34b and MicroRNA-34c are targets of p53 and cooperate in control of cell proliferation and adhesion-independent growth. Cancer Res 67: 8433-8438, 2007.

29. Kanehisa M, Goto S, Sato Y, Furumichi M and Tanabe M: KEGG for integration and interpretation of large-scale molecular data sets. Nucleic Acids Res 40 (Database issue): D109-D114, 2012.

30. Panduri V, Surapureddi S, Soberanes S, Weitzman SA, Chandel N and Kamp DW: P53 mediates amosite asbestos-induced alveolar epithelial cell mitochondria-regulated apoptosis. Am J Respir Cell Mol Biol 34: 443-452, 2006.

31. Cory S and Adams JM: The Bcl2 family: Regulators of the cellular life-or-death switch. Nat Rev Cancer 2: 647-656, 2002.

32. Ouyang YB and Giffard RG: MicroRNAs affect BCL-2 family proteins in the setting of cerebral ischemia. Neurochem Int 77: 2-8, 2014.

33. Loop T, Dovi-Akue D, Frick M, Roesslein M, Egger L, Humar M, Hoetzel A, Schmidt R, Borner C, Pahl HL, et al: Volatile anesthetics induce caspase-dependent, mitochondria-mediated apoptosis in human T lymphocytes in vitro. Anesthesiology 102: 1147-1157, 2005. 